\title{
ON THE ERRORS OF THE EPHEMERIDES DERIVED FROM OPTICAL OBSERVATIONS OF PLANETS
}

\author{
A.S. KHARIN \\ Main Astronomical Observatory \\ Ukrainian Academy of Sciences \\ $252127 \mathrm{Kiev}$ \\ USSR
}

YU.B. KOLESNIK

Astronomical Council

USSR Academy of Sciences

109017 Moscow

USSR

\begin{abstract}
On the basis of about 40000 optical observations of the Sun and major planets obtained with 33 meridian and photographic instruments during last 3 decades a comparative consistency analysis of old and new ephemerides with these observations has been made.

For the inner planets significant improvement in RA is confirmed while in DEC it is less apparent. For outer planets the improvement is strongly marked in DEC for Jupiter and Saturn and espesially for Neptune in both coordinates. Significant systematic differences between meridian and photographic observations are detected.
\end{abstract}

During the last 3 decades a large amount of a newer planetary optical data have been obtained with meridian and photographic methods.

Only a small part of them has been used for creating of a new fundamental ephemeris DE200 [1]. So we have analysed the agreement of all available modern optical observations in the time span 1960-1987 obtained with 19 meridian instruments and 14 astrographs with the old standard ephemerides (Newcomb's theory with Ross corrections for the four inner planets and the EckertBrouwer-Clemence numerical ephemeris for the five outer planets) and with the new one - DE200, adopted as standard by IAU since 1984.

Two homogeneous sets of observation data have been formed by reducing the published values $(\mathrm{O}-\mathrm{C})$ to the corresponding ephemeris and fundamental catalogue. For this purpose the differences between apparent places based on the old and new ephemerides and constants of the IAU were added or subtracted, according to the case, to/from (O-C) for all objects. Fricke's equinox correction [2] was applied in the same way for RA. Systematic differences between reference star catalogues are ignored at this stage of analysis. For each object, method of observation and ephemeris, the curves of normal points in 30 degree zones of orbital longtitude have been obtained. Dispersion of each curve may be interpreted as the combined effect of systematic errors of the ephemeris and observations. The ratio of dispersions of the ephemerides compared should reflect the degree of their relative proximity to the observational data. Cross-correlation of curves will 
display the same systematic errors of both ephemerides. After all computations are made, the results are represented in Table 1 . Values having the significance not exceeding the $5 \%$ level of F-criteria are marked with an asterisk.

Table 1. Ratios of dispersions of normal point curves and cross-correlation coefficients for old and new ephemerides

Ratios of dispersions

$\begin{array}{lll}\text { Objects: } & \text { Meridian } & \text { Photographic } \\ & \text { RA DEC RA DEC }\end{array}$

\begin{tabular}{lcccllllll}
\hline & & & & & & & & & \\
Sun & $11.2^{*}$ & $3.0^{*}$ & - & - & 0.28 & $0.82^{*}$ & - & - \\
Mercury & 2.1 & 1.0 & - & - & $0.65^{*}$ & $0.58^{*}$ & - & - \\
Venus & 2.1 & 1.0 & $8.8^{*}$ & 2.2 & $0.74^{*}$ & $0.87^{*}$ & -0.49 & 0.40 \\
Mars & $3.0^{*}$ & 1.8 & $7.6^{*}$ & $4.3^{*}$ & 0.34 & $0.75^{*}$ & 0.39 & $0.72^{*}$ \\
Jupiter & 1.6 & $13.5^{*}$ & 0.9 & 1.7 & 0.52 & -0.58 & $0.93^{*}$ & -0.24 \\
Saturn & 0.5 & $17.4^{*}$ & 1.3 & $5.3^{*}$ & 0.43 & -0.04 & $0.98^{*}$ & 0.31 \\
Uranus & 2.2 & 1.2 & $12.1^{*}$ & 2.0 & -0.39 & $0.78^{*}$ & $-0.94^{*}$ & $0.93^{*}$ \\
Neptune & $70.9^{*}$ & 0.3 & $123.8^{*}$ & 1.2 & 0.81 & -0.52 & $1.00^{*}$ & $1.00^{*}$ \\
& & & & & & & & & \\
\end{tabular}

Cross-correlation coefficient

Meridian Photographic

RA DEC RA DEC

The examination of this table leads us to the following conclusions:

1. Significant improvement of the new ephemerides for the four inner planets in RA is confirmed by the two methods. An apparent correlation of DEC curves for all these planets is remarkably noticeable while both ephemerides were deviating systematically from observations by approximately 0.5 .

2. As to the 4 outer planets the analysis reveals rather considerable improvement in DEC for Jupiter and Saturn. The same is noticeable for Uranus RA according to photographic data. The very large (about 10" in RA and 1" in DEC) discrepancies of the old Neptune ephemeris are eliminated by using the new one.

3. Some discordance of cross-correlation coefficients-and ratios of dispersions between meridian and photographic measurements-reveal their significant relative systematic differences.

\section{References}

1. Standish, E.M. (1986), in Proc.IAU Symp.114, Relativity in Celest. Mech. and Astrometry, J. Kovalevsky and V.A. Brumberg (eds.), D. Reidel, Dordrecht, 71-83.

2. Fricke, W. (1982), Astron. Astrophys. 107, L13-L16. 